\title{
ON A GENERAL CONVERGENCE FOR BROYDEN LIKE UPDATE METHOD
}

\section{RABINDRANATH SEN, RINI CHATTOPADHYAY and TRIPTI SAHA}

\author{
Department of Applied llathematics \\ Calcutta University \\ 92, A.P.C. Road \\ Calcutta - 700009 \\ India
}

(Receivel December 7, 1987 and in revised form November 20, 1990)

ABSTRACT. The role of Broyden's method as a powerful quasi-Newton method for solving unconstrained optimization problems or a system of nonlinear algebraic equations is well known. We offer here a general convergence criterion for a method akin to Broyden's method in $R^{\prime \prime}$. The approach is different from those of other convergence proofs which are available only for the direct prediction methods.

KEY WORDS AND PHRASES. Unconstrained optimization, Broyden's method, partial ordering in $R^{n}$, M-matrix.

1980 AMS SUBJECT CLASSIFICATION CODE. $65 \mathrm{~K}$.

1. INTRODUCTION.

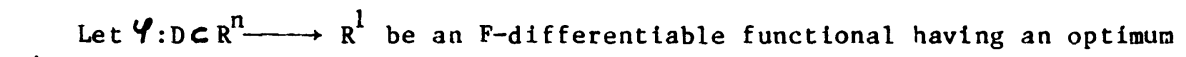
$x^{\star} \epsilon$ int (D).

Then the vector $x^{*}$ at which the optimum of $\varphi$ is realized, satisfies the equation $\nabla \varphi(x)=P(x)=0$

In the above, $\nabla=\left(\frac{\partial}{\partial x_{1}}, \frac{\partial}{\partial x_{2}}, \ldots \frac{\partial}{\partial x_{n}}\right)^{T}$. Our concern in this paper is iterative methods for the solution of simultaneous non-linear equations

$$
F(x)=0 ; F: R^{m} \longrightarrow R^{m}
$$

in the case when the complete computation of $F^{\prime}$ is infeasible.

In the case, $F=P$, solving (1.1) means in effect finding the minimizer of $\varphi$.

The algorithm under consideration takes the form

$$
x_{n+1}=x_{n}-\gamma_{n} H_{n} F\left(x_{n}\right)
$$

where $H_{n}$ is generated by the method in such a way that the quasi-Newton equation

$$
H_{n+1}\left(F\left(x_{n+1}\right)-F\left(x_{n}\right)\right)=x_{n+1}-x_{n}
$$

is satisfied at each step. 
The step length $\gamma_{n}$ is chosen to promote convergence. By analogy with the DFPmethod (Davidson [1], Fletcher and Powell [2]) for unconstrained optimizations and by considering what is desirable when $f$ is linear, Broyden [3] in 1965 suggested an algorithm by which $H_{n+1}$ is obtained froin $H_{n}$ by means of a rank-one update.

In the case of minimizing problems, Dixon [4] called a method perfect if $\gamma_{n}=\gamma_{n}^{*}$ is obtained through line searches and a direct prediction method if $Y_{n}=1$. The iterative procedures $(1.2)$ and (1.3) may be described as follows:

Choose non-singular $\mathrm{H}_{0} \mathrm{R}^{\mathrm{mxm}}$ and also $\mathrm{x}_{\mathrm{o}} \mathrm{R}^{\mathrm{m}}$.

For $n=0,1,2, \ldots$ let $s_{n}=-\gamma_{n} H_{n} F\left(x_{n}\right)$

$\gamma_{n}$ being chosen such that

$$
\begin{aligned}
& \left\|F\left(x_{n+1}\right)\right\| \leqslant\left\|F\left(x_{n}\right)\right\| \\
& x_{n+1}=x_{n}+s_{n} \\
& y_{n}=F\left(x_{n+1}\right)-F\left(x_{n}\right)
\end{aligned}
$$

If $y_{n}=0$ then $\quad H_{n+1}=H_{n}$

and choose $v_{n} R^{m}$ such that $v_{n}^{T} y_{n}=1$ and $v_{n}^{T} H_{n} s_{n} \neq 0$.

Let $H_{n+1}=H_{n}+\left(s_{n}-H_{n} y_{n}\right) v_{n}^{T}$.

Broyden's [3] method (sometimes called his first or good method) results fron choosing $v_{n}=H_{n}^{T} s_{n} / s_{n}^{T} H_{n} y_{n}$ in (1.8) and is defined for $y_{n} \neq 0$ only so long as $s_{n}^{T} H_{n} y_{n} \neq 0$.

Broyden's second or bad method results from choosing $v_{n}=y_{n} / y_{n}^{T} y_{n}$ where $y_{n} \neq 0$ and is defined so long as $y_{n}^{T} H_{n}^{-1} s_{n} \neq 0$.

The convergence results that are avallable to date are proved for the direct prediction method [5]. Broyden has shown that his (first) method converges locally at least linearly on nonlinear problems and at least R-Superlinearly on linear problem [6].

Later Broyden et al [7] showed that both Broyden's good and bad methods converge locally at least Q-superlinearly.

More and Trangstein [8] subsequently proved that 'locally' could be replaced by "globally" when a modified form of Broyden's method 18 applied to linear systems of equations. On the other hand, Gay showed in 1979 [5] that Broyden's good and bad methods enjoy a finite termination property when applied to linear systems with a nonsingular matrix. He has also proved that Broyden's good method enjoys 10 al $2 \mathrm{~m}-\mathrm{step}$ Q-quadratic convergence on non-11near systems.

Recently, Dennis and Walker [9] have made generalizations of their results [7], [10] and have put forward convergence theorems for least-change secant update methods. Decker et al, have considered Broyden's method for a class of problems having singular Jacobian at the root [11].

Our concern is to consider the method (1.2) which is not necessarily a direct prediction method. Our method is not perfect because in the case of minimization problems exact line searches have not been performed. However, the scalars do reduce $\|F(x)\|$ at each step. It may however be noted that perfect methods are not efficient 
[10]. Our method can thus be viewed as an intermediate between direct prediction and perfect methods.

We have called our method Broyden-like because although we have used Broyden's good method we have taken $B_{0}$ to be an M-matrix [12] unlike Broyden [3]. We have used componentwise partial ordering in $R^{m}$ to prove munotone convergence of the sequence $x_{n}$. We have asserted that under certain conditions, $H_{n}$ can be taken as nonnegative matrices. In our analysis, $F$ is taken as an isotone operator [12]. Operators which are monotonically decomposible (MDO) [13] can also be brought within the scope of our convergence theorem. A local linear convergence is achieved. Experiments with numerical problems are also encouraging. Even where Newtun's method has diverged, our method converges in a small number of iterations.

Section 2 contains mathematical preliminaries. Section 3 contains convergence results. Section 4 gives the algorithm. Numerical examples are presented in section 5 while we incorporate some 'discussion' in section 6 .

\section{MATHEMATICAL PREL IMINARIES.}

DEFINITION 2.l. The componentwise partial ordering in $\mathrm{R}^{\mathrm{m}}$ is defined as follows:

$$
\begin{aligned}
& \text { For } x, y \in R^{m}, x=\left(x_{1}, x_{2}, \ldots x_{\text {In }}\right)^{T} \\
& y=\left(y_{1}, y_{2}, \ldots y_{m}\right)^{T}, x>y \text { if and on } 1 y \text { if } \\
& x_{i}>y_{i}, i=1,2, \ldots m, \\
& x>y \Longleftrightarrow x>y \text { and } x \neq y
\end{aligned}
$$

DEFINITION 2.2. We define for any $x, y \in R^{m}$, such that $x<y$, the order interval [11]

$$
\langle x, y\rangle=\left\{u \quad R^{m} / x<u<y\right\} .
$$

DEFINITION 2.3. A mapping $F: D \quad R^{m} \longrightarrow R^{m}$ is isotone (antitone) [12] on $D_{0} D$ if $F x<F y(F x>F y)$ whenever $x \leqslant y, x, y \in D_{0} \cdot$

DEFINITION 2.4. We denote by $L\left(R^{m}\right)$ the space of mxm matrices.

We introduce a partial ordering in $L\left(R^{m}\right)$ which is compatible with the componentwise partial ordering in $\mathrm{R}^{\mathrm{m}}$.

DEFINITION 2.5. A real mxm matrix $\left(a_{i j}\right)$ with $a_{i j}<0$, for all $i \neq j$ is defined to be an M-matrix [12] if $A$ is non-singular and $A^{-1}>0$.

In what follows, $H_{n}=B_{n}^{-1}$, so that $B_{n}$ is a replacement for the Jacobian $J\left(x_{n}\right)$, and thus $B_{n}$ satisfies the quasi-Newton equation

$$
B_{n} s_{n-1}=y_{n-1}, n=1,2, \ldots
$$

According to Broyden's good method, the update formula for $B_{n+1}$ is given by

$$
B_{n+1}=B_{n}-\frac{\left(B_{n} s_{n}-y_{n}\right) s_{n}^{T}}{s_{n}^{T} s_{n}}
$$

In what follows we denote $s_{n}=\left(s_{i}^{(n)}\right), y_{n}=\left(y_{i}^{(n)}\right), B_{n}=\left(b_{i j}^{(n)}\right), 1=1,2, \ldots m$, $j=1,2, \ldots m$.

LEMMA 2.1. (I $\left.-\frac{s_{n} s_{n}^{T}}{s_{n}}\right)$ is a square matrix whose diagonal elements are positive and off-diagonal elements are non-positive provided $s_{i}^{(n)}>0$ and $s^{(n)}>0$ for at least one $i$. 
The proof is trivial.

LEMMA 2.2. Let the following conditions be fulfilled:

(i) $\quad B_{n}=\left(b_{i j}^{(n)}\right), b \underset{i j}{(n)}<0, f, r$ all $i, j, i=j, b_{i i}^{(n)}>0$

(ii) $B_{n}$ is a strictly diagonally dominant matrix

(iii) $\left\{x_{n}\right\}$ is a monotonic increasing sequence in the order sense.

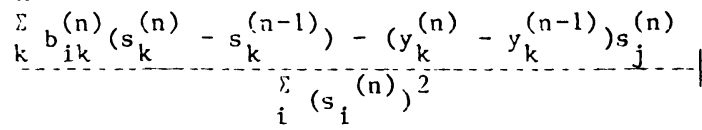

is small compared to $\left|b_{j}^{(n)}\right|$

(v) $\quad \sum_{i \neq j}\left|b_{i j}^{(n)}\right|<b_{i i}^{(n)}+j \sum_{k}\left[b_{i k}^{(n)}\left(s_{k}^{(n)}-s_{k}^{(n-1)}\right)-\left(y_{k}^{(n)}-y_{k}^{(n-1)}\right)\right] s_{j}^{(n)}$

Then $B_{n+1}$ is an M-matrix.

PROOF. It follows fron conditions (i) and ( $i$ ) that $B_{n}$ is an M-matrix [12].

Since $B_{n}$ satisfies the Quasi-Newton equation (2.1)

$$
\left(B_{n} s_{n}-y_{n}\right) s_{n}^{T}=\frac{\left[B_{n}\left(s_{n}-s_{n-1}\right)-\left(y_{n}-y_{n-1}\right)\right] s_{n}^{T}}{s_{n} s_{n}^{T}}
$$

and the $(i, j)^{\text {th }}$ element of $B_{n+1}$ is given by

$$
b_{i j}^{(n)}-\frac{k_{k}\left[b_{i k}^{(n)}\left(s_{k}^{(n)}-s_{k}^{(n-1)}\right)-\left(y_{k}^{(n)}-y_{k}^{(n-1)}\right)\right] s_{j}^{(n)}}{\sum_{j} s_{j}^{(n)}}
$$

since $x_{n}$ is a monotonic increasing sequence $s_{n}=x_{n+1}-x_{n}>0$. In the neighborhood of the solution $x^{*}, s^{(n)}$ will be small and $s^{(n)}-s^{(n-1)}$ will still be smaller in magnitude. $s_{j}^{(n)} / \sum_{j}^{S}\left(s_{j}^{(n)}\right)^{2} \quad$ will also have at most a finite magnitude.

If $b_{i j}^{(n)} \neq 0$, $i \neq j$, the signs of $b_{i j}^{(n)}$ will determine the signs of $b_{i j}^{(n+1)}$.

Therefore the diagonal elements of $b_{i j}^{(n+1)}$ will be strictly positive and the offdiagonal elements strictly negative. Since $B_{n}$ is a strictly diagonally dominant matrix the strict diagonal dominance property of $B_{n+1}$ will be maintained by virtue of condition (v).

Let us denote

by $c_{i j}^{(n)}$.

$$
\frac{\sum_{k}\left[b_{i k}^{(n)}\left(s_{k}^{(n)}-s_{k}^{(n-1)}\right)-\left(y_{k}^{(n)}-y_{k}^{(n-1)}\right)\right] s_{j}^{(n)}}{\sum_{j}\left(s_{j}^{(n)}\right)^{2}}
$$

Condition (v) implies that

$$
\sum_{i \neq j}^{j}-\left(b_{i j}^{(n)}+c_{i j}^{(n)}\right)<b_{i i}^{(n)}+c_{i i}^{(n)}
$$

and since $\left|b_{i j}^{(n)}\right|$ is large compared to $\left|c_{i j}^{(n)}\right|,(2.4)$ implies that

$$
\dot{i}_{j}\left|b_{i j}^{(n+1)}\right|<\left|b_{i i}^{(n+1)}\right|
$$


REMARK 2.1. If however $B_{n+1}$ ceases to be an M-matrix, we can without violating the constraint (2.1), pre-multiply the first term in the expression for $B_{n+1}$ by a strictly positive diagonal matrix $P_{n}$ of the same order of $B_{n}$, such that $P_{n} B_{n}\left(I-\frac{s_{n} s_{n}^{T}}{s_{n}^{T}}\right)$ becomes the predominant term in the expression for $\widetilde{B}_{n+1}$ as given below.

$$
\widetilde{B}_{n+1}=P_{n} B_{n}\left(I-\frac{s_{n} s_{n}^{T}}{s_{n}^{T} s_{n}}\right)+\frac{y_{n} s_{n}^{T}}{s_{n}^{T} s_{n}} .
$$

Thus, if $B_{n}$ is already a strictly diagonally dominant matrix with $b_{i j}^{(n)}<0, i \neq j$ and $b_{i j}^{(n)}>0, B_{n+1}$ will also be a strictly diagonally dominant matrix with $b_{i j}^{(n+1)}<0$, $i \neq j$, and $b_{i i}^{(n+1)}>0$. Therefore, if $B_{n}$ is an M-matrix we can always construct $B_{n+1}\left(=\breve{B}_{n+1}\right)$ as an M-matrix without affecting the condition $B_{n} s_{n}=y_{n}$. Writing $B_{n}^{-1}=H_{n}$ and $B_{n+1}^{-1}=H_{n+1}$, we obtain from (2.5)

$$
\begin{aligned}
H_{n+1} & =\left[P_{n} B_{n}\left(I-\frac{s_{n} s_{n}^{T}}{s_{n}^{T} s}+\frac{B_{n}^{-1} P^{-1} y_{n} s_{n}^{T}}{s_{n}^{T} s_{n}}\right)\right]^{-1} \\
& =\left[I-\frac{s_{n} s_{n}^{T}}{s_{n}^{T} s_{n}}+\frac{B_{n}^{-1} P_{n}^{-1} y_{n} s_{n}^{T}}{s_{n}^{T} s_{n}}\right]^{-1} B_{n}^{-1} P_{n}^{-1}
\end{aligned}
$$

which is approximately equal to

$$
\left[I+\left(s_{n}-H_{n} P_{n}^{-1} y_{n}\right) \frac{s_{n}^{T}}{s_{n}^{T} s_{n}}\right] H_{n} P_{n}^{-1}
$$

Writing $s_{n}=\widetilde{B}_{n+1}^{-1} y_{n}=H_{n+1} y_{n}$, which is approximately equal to $H_{n} P_{n}^{-1} y_{n}$, $H_{n+1}$ can be taken as

$$
H_{n+1}=H_{n} P_{n}^{-1}+\left(s_{n}-H_{n} P_{n}^{-1} y_{n}\right) \frac{s_{n}^{T} H_{n} P^{-1}}{s_{n}^{T} H_{n} P_{n}^{-1} y_{n}}
$$

Therefore, $\mathrm{H}_{n+1}$ as expressed by (2.6) satisfles the Quasi-Newton equation $\mathrm{H}_{\mathrm{n}+1} \mathrm{y}_{\mathrm{n}}=\mathbf{s}_{\mathrm{n}}$.

LEMMA 2.3. Let the diagonal elements of $b_{1 j}^{(n)}$ of the strictly diagonally dominant matrix $B_{n}$ satisfy the condition $\left|b_{1 i}^{(n)}\right|<M$, for all 1 , where $M(>0)$ is a finite constant. Then

$$
\frac{1}{\left|\lambda_{i}^{(n)}\right|}>\frac{1}{2 n}, \lambda_{i}^{(n)} \text { are the eigenvalues of } B_{n} \cdot
$$

PROOF. By the Greschgorin theorem,

$$
\left|\lambda_{i}^{(n)}-b_{i i}^{(n)}\right|<\sum_{i \neq j}\left|b_{i j}^{(n)}\right| \text {. }
$$

By strict diagonal dominance of $B_{n}$,

$$
\left|\lambda_{i}^{(n)}\right|<2\left|b_{i 1}^{(n)}\right|<2 M .
$$


Therefore,

$$
\left|\lambda_{i}^{(n)}\right| \bar{l} \mid \frac{1}{2 M}>0 \text {. }
$$

The above lemma ensires that the lower bound of the eigenvalues of $H_{n}=B_{n}^{-1}$ are strictly positive Eor all $n$ and hence ${ }_{n}$ remains nonsingilar at each iteration. The stability of the iteration process is therefore guaranteed.

In what follows $\|$.$\| denotes an arbitrary norn in R^{m}$ and the operator norm is induced by the particilar vector norm.

3.1. CONVERGENCE.

THEOREM 3.1. Let the following conditions be fulfilled:

(i) $\quad F: D \subset R^{m} \longrightarrow R^{m}$ is Frechet differentiable on a convex set $D_{0} \subset D$.

(ii) $D_{0}$ includes the null element and $x_{0}, y_{0} \in D_{0}$ such that $i f x \leqslant y$ then the srder interval $\langle x, y\rangle C D_{0}$.

(iii) $F(x) \leqslant 0$, for all $x D_{0}$ and $F$ is isotone i.e. if $x<y, F(x) \leqslant F(y)$ for a $L 1 x, y \in D_{0}$.

(iv) $x_{0} \in D_{0}$ is an initial approximation to the solution and $x_{0}>0$ and $F\left(x_{0}\right)<0$.

(i) The operator $G=I-\gamma_{0} H_{0} F$ is such that $G D_{0} \subset D_{0}$.

(vi) The operator $B_{0}=\left(b_{i j}^{(o)}\right)$ is a strictly diagonally doninant matrix with $b_{i j}^{(0)}<0$, for all $i, j, i \neq j$, and $b_{i i}^{(o)}>0$.

(vii) For $B_{n}=b_{i j}^{(n)}$

$$
\frac{\left|b_{k}^{\sum}\left[b_{i k}^{(n)}\left(s_{k}^{(n)}-s_{k}^{(n-1)}\right)-\left(y_{k}^{(n)}-y_{k}^{(n-1)}\right)\right] s_{j}^{(n)}\right|}{\sum_{i}\left(s_{i}^{(n)}\right)^{2}}
$$

is small compared to $\left|b_{i j}^{(n)}\right|, n=0,1,2, \ldots$

$$
\begin{aligned}
& \text { (viii) } \sum_{\substack{j \neq j \\
j}} b_{i j}^{(n)}<b_{i i}^{(n)}+\frac{\sum_{i} b_{i k}^{(n)}\left(s_{k}^{(n)}-s_{k}^{(n-1)}\right)-\left(y_{k}^{(n)}-y_{k}^{(n-1)}\right) s_{j}^{(n)}}{\sum_{i}\left(s_{i}^{(n)}\right)}, \\
& n=0,1,2, \ldots \\
& \text { (ix) }\left|b_{i i}^{(n)}\right| \leqslant(k>0), n=0,1,2, \ldots .
\end{aligned}
$$

(x) The scalars $\gamma_{n}(>\alpha>0)$ are to be chosen such that

(a) $\quad Y_{n-1}{ }_{n-1}^{H} F\left(x^{0}\right) \leqslant \gamma_{n}{ }_{n} F\left(x_{0}\right)<0, n>1$

(b) $\sup _{(x)}\left[I-\gamma_{n} H_{n} F^{\prime}(x)\right] \leqslant\left[I-\gamma_{n-1} H_{n-1} F^{\prime}\left(x_{n-1}\right)\right], x \in\left\langle x_{n-1}, x_{n}\right\rangle$

(xi) $F^{\prime}(x)$ is Frechet differentiable for all $x \in D_{0}$. 
(xii) $\lim _{n \rightarrow \infty}\left[I-Y_{0} H_{0} F^{\prime}\left(x_{0}\right)\right]^{n}\left[x_{1}-x_{0}\right]=0$ in the order sense.

Then starting $f$ roin $x_{0}>0$, an initial approximation to the solution of $F(x)=0$ is the sequence $\left\{x_{n}\right\}$ of Broyden-1ike approximations defined by

$$
x_{n+1}=x_{n}-\gamma_{n}^{H}{ }_{n} F\left(x_{n}\right), n=0,1, \ldots
$$

which converges to a solution of the equation $F(x)=0$.

If in particular, it is possible to find a positive convergent matrix A, i.e., $A^{n}+0$ as $n \rightarrow \infty$, then the error at the $n t h$ stage 1 s given by

$$
\left\|x^{*}-x_{n}\right\|<\left\|A^{n}(I-A)^{-1}\left(x_{1}-x_{0}\right)\right\|
$$

PROOF. Conditions (iii), (iv) and ( $x a)$ yield

$$
x_{1}=x_{0}-\gamma_{0} H_{0} F\left(x_{0}\right)>x_{0}>0
$$

By $(v), 0<x_{1} \in D_{0} C D$. Hence $G x_{1} \in D_{0}$.

$B_{0}$ is an M-matrix [12] by (vi). Hence $H_{0}=B_{0}^{-1}>0, s_{0}=x_{1}-x_{0}>0$.

Because of conditions ( $v i),(v i i)$ and ( $i i i)$ and because $s_{0}>0$, it follows from (2.2) that $B_{1}$ is an M-matrix. Therefore, $\mathrm{H}_{1}=\mathrm{B}_{l}^{-1}>0$.

Isotonicity of $F$, together with condition ( $x a$ ) yield,

$$
0>\gamma_{1} H_{1} F\left(x_{1}\right)>\gamma_{1} H_{1} F\left(x_{0}\right)>\gamma_{0} H_{0} F\left(x_{0}\right) \text {. }
$$

Hence $x_{2}>x_{1}>0$. Thus $x_{1} \leqslant x_{2}=x_{1}-\gamma_{0} H H_{0} F\left(x_{0}\right)=G x_{1} \in D_{0}$.

Let us assume by way of induction that $0<x_{k} \in D_{0}, k=1,2, \ldots n$, and $B_{k}$ is an $M-$ matrix, $k=1,2, \ldots n$, with $b_{i j}^{(k)}<0, i \neq j$.

Since $B_{n}$ is an $M$-matrix, $H_{n}>0$,

By conditions (iii) and ( $x a) \gamma_{n} H_{n} F\left(x_{n}\right)>\gamma_{n} H_{n} F\left(x_{0}\right)$

$$
\begin{aligned}
& >\ldots . . . \\
& >\gamma_{0} H_{0} F\left(x_{0}\right)
\end{aligned}
$$

Therefore, $x_{n}<x_{n+1}<x_{n}-Y_{n} H_{n} F\left(x_{0}\right)$

$$
\begin{aligned}
& <x_{n}-\gamma_{0} H_{0} F\left(x_{0}\right) \\
& =G x_{n} \in D_{0}
\end{aligned}
$$

Using the fact that $B_{n}$ is an M-matrix with $b_{i j}^{(n)}<0, i \neq j, x_{n}<x_{n+1}$, and the conditions (vii) and (viii), we can conclude from Lemma (2.2) that $B_{n+l}$ is an $M-m a t r i x$ with $b_{i j}^{(n+1)}<0, i \neq j$.

Therefore the induction is completed.

Thus, $\left\{x_{k}\right\}$ is a monotonic increasing sequence in the order sense and $x_{k} \in D_{o}$, for al1 k.

$$
\text { Now, } \begin{aligned}
0 \leqslant x_{2}-x_{1}= & x_{1}-x_{0}-\gamma_{1} H_{1}\left[F\left(x_{1}\right)-F\left(x_{0}\right)\right] \\
& -\left[\gamma_{1} H_{1} F\left(x_{0}\right)-\gamma_{0} H_{0} F\left(x_{0}\right)\right] \\
& <x_{1}-x_{0}-\gamma_{1} H_{1}\left[F\left(x_{1}\right)-F\left(x_{0}\right)\right] .
\end{aligned}
$$


$D_{0}$ being a convex set and using the mean value theorem in $R^{m}$ we obtain from (3.5)

$$
x_{2}-x_{1}<\int_{0}^{1}\left[I-r_{1} H_{1} F^{\prime}\left(x_{0}+t\left(x_{1}-x_{0}\right)\right)\right]\left(x_{1}-x_{0}\right) d t \quad 0<t<1
$$

Here' stands for differentiation in the Frechet sense.

Again since $F^{\prime}\left(x_{0}+t\left(x_{1}-x_{0}\right)\right), 0<t<1$ is $G$ differentiable, $F^{\prime}\left(x_{0}+t\left(x_{1}-x_{0}\right)\right)$ is semi-continuous [12].

Therefore, the operator $G_{1}(t)=F^{\prime}\left(x_{0}+t\left(x_{1}-x_{0}\right)\right)$ is continuous in $t[0,1]$. We thus have the following from (3.6)

$$
\begin{aligned}
0 \leqslant x_{2}-x_{1} & \leqslant \sup _{t}\left[I-\gamma_{1} H_{1} F^{\prime}\left(x_{0}+t\left(x_{1}-x_{0}\right)\right)\right]\left(x_{1}-x_{0}\right) \\
& =\left[I-\gamma_{1} H_{1} F^{\prime}\left(x_{0}+t\left(x_{1}-x_{0}\right)\right)\right]\left(x_{1}-x_{0}\right)
\end{aligned}
$$

assuming the supremum is attained at $t=t, 0<t<1$.

By $(x b),(3.7)$ further simplifies to

$$
0 \leqslant x_{2}-x_{1} \leqslant\left[I-\gamma_{0} H_{0} F^{\prime}\left(x_{0}\right)\right]\left(x_{1}-x_{2}\right) \text {. }
$$

Arguing analogously as before,

$$
\begin{aligned}
x_{n+1}-x_{n} & \leqslant \int_{0}^{1}\left[\left(I-\gamma_{n} H_{n} F^{\prime}\left(x_{n-1}+t\left(x_{n}-x_{n-1}\right)\right)\right]\left(x_{n}-x_{n-1}\right) d t\right. \\
& \leqslant \sup _{t}\left[I-\gamma_{n} H_{n} F^{\prime}\left(x_{n-1}+t\left(x_{n}-x_{n-1}\right)\right)\right]\left(x_{n}-x_{n-1}\right) \\
& =\left[I-\gamma_{n} F_{n} F^{\prime}\left(x_{n-1}+\tilde{t}\left(x_{n}-x_{n-1}\right)\right)\right]\left(x_{n}-x_{n-1}\right)
\end{aligned}
$$

assuming that the supremum is attained at $t=\tilde{t}$.

Condition $(x b)$ further reduces (3.9) to

$$
\begin{aligned}
x_{n+1}-x_{n} & \leqslant\left[I-\gamma_{n-1} H_{n-1} F^{\prime}\left(x_{n-1}\right)\right]\left(x_{n}-x_{n-1}\right) \\
& \leqslant\left[I-\gamma_{0} H_{0} F^{\prime}\left(x_{0}\right)\right]\left(x_{n}-x_{n-1}\right) \\
& \leqslant\left[I-\gamma_{0} H_{0} F^{\prime}\left(x_{0}\right)\right]^{n}\left(x_{1}-x_{0}\right) .
\end{aligned}
$$

Hence $x_{n+p}-x_{n} \leqslant \sum_{k=n}^{n+p-1}\left[I-\gamma_{0} H_{0} F^{\prime}\left(x_{0}\right)\right]^{k}\left(x_{1}-x_{0}\right)$

Since $\left[I-\gamma_{0} H_{0} F^{\prime}\left(x_{0}\right)\right]^{n}\left(x_{1}-x_{0}\right)+0$ as $n \rightarrow \infty$ it follows from ( $\left.x i l\right)$ that $\left\{x_{n}\right\}$ is a Cauchy sequence and the space is complete,

$$
x^{*}=\lim _{n \rightarrow \infty} x_{n} R^{m}
$$

Using convergence of $\left\{x_{n}\right\}$ it follows from (3.12) that

$$
\lim _{n \rightarrow \infty} \gamma_{n} H_{n} F\left(x_{n}\right)=0 \text {. }
$$

Now, $F\left(x_{n}\right)=\frac{1}{Y_{n}} B_{n}\left(x_{n+1}-x_{n}\right)$.

Therefore using $(i x),(x)$ and the strict diagonal dominance of $B_{n}$

$$
\left\|F\left(x_{n}\right)||<\frac{2 k}{\alpha}\right\| x_{n+1}-x_{n} \| \rightarrow 0 \text { as } n+\infty \text {. }
$$

Continuity of $F$ yields that

$$
F\left(x^{*}\right)=0 \text {. }
$$

Hence $x^{*}$ is a solution of the equation $F(x)=0$.

Further if $\mathrm{I}-\mathrm{Y}_{\mathrm{O}} \mathrm{H}_{\mathrm{O}} \mathrm{F}^{\prime}\left(\mathrm{x}_{\mathrm{O}}\right)<\mathrm{A}$, (3.11) reduces to 


$$
x_{n+p}-x_{n}<\sum_{k=n}^{n+p-1} A^{k}\left(x_{1}-x_{0}\right)
$$

The error (3.2) follows from (3.15) by making $p+\infty$ and then taking $\|\cdot\|$.

In the next theorem we provide a bound for the inverse Jacobian approximation.

THEOREM 3.2. Let in addition to the conditions of theorem (3.1), the following conditions be satisfied:

(a) $\left\|F^{\prime}(x)-F^{\prime}(y)\right\| \leqslant \gamma^{\prime}\|x-y\|$, for all $x, y \in D_{0}$

(b) $\left[F^{\prime}\left(x_{0}\right)\right]^{-1}$ exists and $\left\|\left[F^{\prime}\left(x_{0}\right)\right]^{-1}\right\|<B^{\prime}$

(c) $\quad\left\|\sum_{n=0}^{\infty}\left(I-\gamma_{0} H_{0} F^{\prime}\left(x_{0}\right)\right)^{n}\left(x_{1}-x_{0}\right)\right\|<B^{\prime} \gamma^{\prime}$

Then

$$
\left\|\left[F^{\prime}\left(x_{n}\right)\right]^{-1}-\gamma_{n} H_{n}\right\|<\frac{\left\|I-\gamma_{0} H_{0} F^{\prime}\left(x_{0}\right)\right\|}{1-B^{\prime} \gamma^{\prime}\left\|\left.\right|_{k=0} ^{n-1}\left(I-\gamma_{0} H_{0} F^{\prime}\left(x_{0}\right)\right)^{k}\left(x_{1}-x_{0}\right)\right\|}
$$

PROOF. Following Rheinboldt and Ortega [12] we show that $[F(x)]^{-1}$ exists at all the iteration points.

Let $\underline{\Omega}\left(x_{0},\left(\beta^{\prime} \gamma^{\prime}\right)^{-1}\right)$ denote the closed sphere with $x_{0}$ as the center and $\left(\beta^{\prime} \gamma^{\prime}\right)^{-1}$ as the radius.

$$
\text { Let } D_{1}=\underline{\Omega}\left(x_{0},\left(B^{\prime} \gamma^{\prime}\right)^{-1}\right) \cap D_{0}
$$

Then, for $x \in D_{1}$ we have

$$
|| F^{\prime}(x)-F^{\prime}\left(x_{0}\right)\left\|<\gamma^{\prime}|| x-x_{0}\right\|<1 / \beta^{\prime}
$$

Now,

$$
\begin{aligned}
& \left\|I-\left[F^{\prime}\left(x_{0}\right)\right]^{-1} F^{\prime}\left(x_{1}\right)\right\| \\
& \|\left[\left(F^{\prime}\left(x_{0}\right)\right]^{-1}\left[F^{\prime}\left(x_{0}\right)-F^{\prime}\left(x_{1}\right)\right] \|<B^{\prime} \frac{1}{B^{\prime}}=1\right.
\end{aligned}
$$

Therefore $\left[F^{\prime}\left(x_{0}\right)\right]^{-1} F^{\prime}\left(x_{1}\right)$ has an inverse and hence for $x \in D_{1}, F^{\prime}(x)$ has an inverse. Moreover, for $x \in D_{1}$, using the Neumann Lemma we get

Therefore,

$$
\left[F^{\prime}(x)\right]^{-1}=\sum_{n=0}^{\infty}\left(\left[F^{\prime}\left(x_{0}\right)\right]^{-1}\left[F^{\prime}\left(x_{0}\right)-F^{\prime}(x)\right]\right)^{n}\left[F^{\prime}\left(x_{0}\right)\right]^{-1}
$$

$$
\begin{aligned}
& \| \sum_{n=0}^{\infty}\left(\left[\left(F^{\prime}\left(x_{0}\right)\right]^{-1}\left[F^{\prime}\left(x_{0}\right)-F^{\prime}\left(x^{\prime}\right]\right)^{n} \|\right.\right. \\
& <\sum_{n=0}^{\infty}\left(B^{\prime} \gamma^{\prime}\left\|x-x_{0}\right\|\right)^{n}=\frac{1}{1-\beta^{\prime} \gamma^{\prime}}\left\|x-x_{0}\right\|
\end{aligned}
$$

since $B^{\prime} y^{\prime}\left\|x-x_{0}\right\|<1$

Therefore for $x \in D_{1}$

$$
\left\|\left[F^{\prime}(x)\right]^{-1}\right\|<\frac{1-\beta^{\prime} \gamma^{\prime}}{\left\|x-x_{0}\right\|}
$$

$\left\{x_{n}\right\}$ being a monotonic increasing sequence it follows from (3.10) and condition (c) of Theorem (3.2),

$$
\left\|x_{n}-x_{0}\right\|<\left\|\left.\right|_{k=0} ^{n-1}\left(I-\gamma_{0} H_{0} F\left(x_{0}\right)\right)^{k}\left(x_{1}-x_{0}\right)\right\|<B^{\prime} \gamma^{\prime}
$$

Hence $\quad x_{n} \in \underline{\Omega}\left(x_{0}, B^{\prime} \gamma^{\prime}\right) \cap D_{0}=D_{1}$

Utilizing condition $(x b)$ and $(3.21)$ we get 


$$
\begin{aligned}
& \left\|\left[F^{\prime}\left(x_{n}\right)\right]^{-1}-\gamma_{n}{ }_{n}{ }_{n}\right\|=\|\left[I-\gamma_{n} H_{n} F^{\prime}\left(x_{n}\right) \mid\left[F^{\prime}\left(x_{n}\right)\right]^{-1} \|\right. \\
& \leqslant\left\|I-\gamma_{n}{ }_{n}^{H} F^{\prime}\left(x_{n}\right)\right\|\left\|\left[F^{\prime}\left(x_{n}\right)\right]^{-1}\right\| \\
& \leqslant \frac{1-\beta^{\prime} \gamma_{1}|| x_{n}-x_{0} \|}{B^{\prime}\left\|I-r_{0} H_{0} F^{\prime}\left(x_{0}\right)\right\|}
\end{aligned}
$$

Using (3.10) and the monotonic increasing property of $\left\{x_{n}\right\}$ we further conclude that

$$
\left\|\left[F^{\prime}(x)\right]^{-1}-\gamma_{n} H_{n}\right\| \leqslant \frac{B^{\prime}\left\|I-\gamma_{0} H_{0} F^{\prime}\left(x_{0}\right)\right\|}{1-B^{\prime} \gamma^{\prime} \|\left.\right|_{k=0} ^{n} \sum_{0}^{1}\left[I-\gamma_{0} H_{o} F^{\prime}\left(x_{0}\right)\right]^{-k^{-}}\left(x_{1}-x_{o}\right)} \|
$$

THEOREM 3.3. If condition $(x b)$ of Theorem 3.1 is replaced by the following condition:

$$
\begin{aligned}
\left(I-\gamma_{n-1} H_{n-1} F^{\prime}\left(x_{n-1}\right)\right)> & \alpha^{\prime} \sup \left(I-\gamma_{n} H_{n} F^{\prime}\left(x_{n}\right)\right) \\
& a^{\prime}>1, D_{n}>1
\end{aligned}
$$

and all other conditions of theorem 3.1 are fulfilled then the superlinear convergence of the sequence $\left\{x_{n}\right\}$ is ensured.

$$
\text { PŔOOF. } \begin{aligned}
\lim _{n \rightarrow \infty}\left(I-Y_{n} H_{n} F^{\prime}\left(x_{n}\right)\right) & \leqslant \sup \left(I-\gamma_{n} H_{n} F^{\prime}\left(x_{n}\right)\right) \\
& x D_{0} \\
& \leqslant \frac{1}{\alpha^{n}}\left(I-\gamma_{0} H_{0} F^{\prime}\left(x_{0}\right)\right),\left(\alpha^{\prime}>1\right) \\
& +0 \text { as } n+\infty .
\end{aligned}
$$

If

$$
\begin{aligned}
& \lim _{n \rightarrow \infty} x_{n}=x^{*} \text { then } \\
& 0<x^{*}-x_{n-1}=x^{*}-x_{n}-Y_{n}{ }_{n}\left[F\left(x^{*}\right)-F\left(x_{n}\right)\right] \\
& =\int_{0}^{1}\left[I-Y_{n}{ }_{n} F^{\prime}\left(x_{n}+t\left(x^{*}-x_{n}\right)\right)\right]\left(x^{*}-x_{n}\right) d t .
\end{aligned}
$$

By arguments analogous to theorem 3.1

$$
\left\|x^{*}-x_{n+1}\right\|<\sup _{t \in[0,1]}\left\|I-\gamma_{n}^{H} F^{\prime}\left(x_{n}+t\left(x^{*}-x_{n}\right)\right)\right\|\left\|x-x_{n}\right\| .
$$

Therefore,

$$
\frac{\left\|x^{*}-x_{n+1}\right\|}{\left\|x^{*}-x_{n}\right\|} \sup _{t \in[0,1]}\left\|I-\gamma_{n} H_{n} F^{\prime}\left(x_{n}+t\left(x^{*}-x_{n}\right)\right)\right\|, .
$$

The continuity of $F^{\prime}(x)$ for $x \in D_{0}$ and relation (3.25) yield,

$$
\lim _{n \rightarrow \infty} \frac{\left\|x^{*}-x_{n+1}\right\|}{\left\|x^{*}-x_{n}\right\|}<\lim \left(I-\gamma_{n \rightarrow \infty} H_{n} F^{\prime}(x)\right)=0
$$

This proves superlinear convergence of $\left\{x_{n}\right\}$.

REMARK 3.1. It may be noted that conditions (vii) and (viii) of theorem 3.1 are required only to prove that $B_{n+1}$ is an $M$-matrix provided $B_{n}$ is so. The question may be raised as to how one can know these conditions in advance. From computational experience one can say that such conditions are usually satisfied. If that is not so, we have indicated in remark 2.1 how $B_{n+1}$ can be made an $M$-matrix when $B_{n}$ is so.

\section{ALGORITHM.}

Step 1. Find $D_{0}: \underset{\sim}{x}<x<\tilde{x}$ in which $F(x)$ is isotone. 
Step 2. Choose $x_{0} \in D_{0}$ and $x_{0}>0$ s.t. $F\left(x_{0}\right)<0$

Step 3. Choose $H_{0}>0, \gamma_{0}=1$ and compute $x_{1}, k=0$.

Step 4. Compute $A_{0}=I-\gamma_{0} H_{0} F^{\prime}\left(x_{0}\right)$.

If $\left(I-\gamma_{0} H_{0} F^{\prime}\left(x_{0}\right)\right)^{n}\left(x_{1}-x_{n}\right) \rightarrow 0$ for some $n>n_{0}$ go to step 5 , otherwise go to step 3 .

Step 5. Compute $s_{k}=-\gamma_{k} H_{k} F\left(x_{k}\right), x_{k+1}=x_{k}+s_{k}$ and $F\left(x_{k+1}\right)$

Step 6. If $F\left(x_{k+1}\right) \simeq 0\left(10^{-4}\right)$ stop, otherwise go to step 7 .

St ep 7. Compute $y_{k}=F\left(x_{k+1}\right)-F\left(x_{k}\right), s_{k}^{T} H_{k} y_{k}$

If $s_{k}^{T} H_{k} y_{k}=0, H_{k+1}=H_{k}$ go to step 8 .

Ot herwise $H_{k+1}=H_{k}+\left(s_{k}-H_{k} y_{k}\right)\left[\frac{H_{k}^{T} s_{k}}{s_{k}^{T} H_{k} y_{k}}\right]^{T}$

Step 8. Compute $\gamma_{k+1}$ s.t. $\gamma_{k+1} H_{k+1} F\left(x_{0}\right)>\gamma_{k} H_{k} F\left(x_{0}\right)$

Step 9. If I $-\gamma_{k} H_{k} F^{\prime}\left(x_{k}\right)>\sup _{x \in\left\langle x_{k}, x_{k+1}\right\rangle}\left[I-\gamma_{k+1} H_{k+1} F^{\prime}(x)\right]$ go to step 10, otherwise go to step 8 .

St ep 10. If $k=k+1$, go to step 5 .

REMARK 4.1. (i) The implementation of the conditions ( $x a$ ) and ( $x b$ ) for the determination of the scalar $\gamma_{n}$ can be done by a computer. (ii) The matrix $P_{n}$ in note (2.1) is arbitrary except that the elements of $P_{n}$ are greater than $p_{n}(>0)$. This must lead to some arbitrariness in $\mathrm{H}_{n+l}$. In that case the choice of $\gamma_{n+1}$ covertly depends on $P_{n}$ in addition to the conditions of $(x a)$ and $(x b)$. $H_{n+1}$ so generated is however a solution of the Quasi-Newton equation. (iii) The total number of multiplications and divisions in $f$ inding $x_{k+1}, H_{k+1}$ and $\gamma_{k+1}$ respectively is $\left(m+m^{2}\right), 2 m^{2}+m, 2 m^{2}+3 m$, i.e. $5 m^{2}+5 \mathrm{~m}$. (iv) The number of function evaluations is $\mathrm{m}^{2}+2 \mathrm{~m}$.

\subsection{NUMERICAL EXAMPLE.}

We take an example [14] in which every equation is linear except for the last equation which is highly non-linear. We choose

where

$$
F(x)=\left[f_{i}(x)\right]^{T}, i=1,2, \ldots N-1
$$

$$
f_{1}(x)=-(N+1)+2 x^{i}+\sum_{\substack{j=1 \\ i \neq j}}^{n} x^{j}, 1=1,2 \ldots N-1
$$

and

$$
f_{N}(x)=-1+\prod_{j=1}^{N} x^{j}
$$

The problem was run for $N=5,10$ and 30 . We take $x_{0}^{1}=0.5$ so that $F\left(x_{0}\right)<0$. Incidentally $F(x)$ is isotone.

Define $D_{0}$, the rectangular parallelepiped given by $0.5 \leqslant x^{1} \leqslant 1.0,1=1,2, \ldots N$, and choose $\gamma_{0}=1 . H_{0}=\left(h_{i j}^{0}\right)$ where $h_{i j}^{0}=0.1,1 \neq N, h_{N N}^{0}=0.5 . h_{i j}=.01$. $A=\left[I-\gamma_{0} H_{0} F^{\prime}\left(x_{0}\right)\right]$ is a convergent matrix.

We summarize our computational experience in table 1. The computations were performed on a Burroughs computer at the R.C.C. Calcutta using a FORTRAN IV language. We solved the problem and in each case the exact solution was obtained. In 
table 2 we provide a comparison of our results with those of Newton's method and Brown's method. For computational results of Newton's method and Brown's method see [14].

Table 1

\begin{tabular}{ccccc}
\hline Dimension $(N)$ & No. of iterations $(n)$ & $\mathrm{x}(\mathrm{x})$ & CPU Time \\
\hline 5 & 5 & $(1,1,1)^{\mathrm{T}}$ & 0 & $3.637 \mathrm{sec}$ \\
10 & 5 & $(1,1, \ldots, 1)^{\mathrm{T}}$ & 0 & $4.462 \mathrm{sec}$ \\
30 & 6 & $(1,1, \ldots, 1)^{\mathrm{T}}$ & 0 & $8.548 \mathrm{sec}$
\end{tabular}

Table 2

\begin{tabular}{clll}
\hline Dimension(N) & Newton's method & Brown's Method & Broyden-1ike method \\
5 & Converged in 18 its & Converged in 6 its & Converged in 5 its \\
10 & Diverged $\|\left. x\right|_{\ell} ^{l} \sim 10^{3}$ & Converged in 9 its & Converged in 5 its \\
30 & Diverged $\|x\|_{l_{2}}^{1} \sim 10^{6}$ & Converged in 9 its & Converged in 6 its
\end{tabular}

Although Brown's method has taken a larger number of tterations, tt may take less CPU time than that of the Broyden-1ike method because Brown's method is quadratically convergent.

\section{DISCUSSIONS.}

(i) In the case of Broyden's method the initial estimate $B_{0}$ is found by taking a finite difference analogue of $F^{\prime}\left(x_{0}\right)$. We are considering the casp where the complete computation of $F^{\prime}(x)$ is infeasible. In our Broyden-like method we start with $B_{0}$ an $M-$ matrix so that $\mathrm{H}_{0}>0$. Since $\mathrm{F}$ is an isotone mapping 1 t could be that all the entries of $F^{\prime}\left(x_{0}\right)$ are non-negative. Nevertheless we can choose $B_{0}$ such that $\left\|B_{0}-F^{\prime}\left(x_{0}\right)\right\|^{0}$ is sufficiently small. Such a $B_{0}$ satisfies the Quasi-Newton equation. Mreover we have used Broyden updates (good method) and as such we have called our method the Broyden-like method. (i1) In the case of DFP's method, $H_{0}$ is always taken as a symmetric positive-definite matrix. Moreover, a symmetric M-matrix is positive definite [12]. Hence our $H_{n}$ can sometimes turn out to be a positvedefinite matrix as in DFP's method. (1ii) Theorem 3.1 can only provide us with nonnegative solutions of nonlinear equations. With a suitable translation we can transform the given equation into another equation having non-negative solutions only. (iv) Our convergence proof seems to be much more elegant than the cases where "majorization principle" has been utilized or where the Euclidean norm of $E_{i}\left(E_{i}=A^{-1} B_{i}-I\right.$, for linear system of equations $\left.A_{x}=b\right)$ has been utilized. (v) The convergence theorem 3.1 is applicable where $F$ is isotone. This restricts the sphere of applicability of the theorem. However, in a large number of problems, the 
nonlinear operators are Monotonically Decomposible (MDO) [13] and convergence theorems along the line of theorem 3.1 can be developed for such operators. The result would be conmunicated in a separate paper.

ACKNOWL EDGEMENTS.

The authors are extremely grateful to Dr. L. Dixon of Hatfield Polytechnic U.K. for some constructive comments.

The second author, who is a N.B.H.M (India) research awardee, is grateful to the National Board for Higher Mathematics, India for Einancial support to carry out the research.

\section{REFERENCES}

1. DAVIDON, W.C., Variable metric method for minimization. Argonne Nat. Labs. Report ANL-5990, Rev. Argonne II (1959).

2. FLETCHER, R. and POWELL, M.J.D., A rapidly convergent descent method for minimizations. Comput J. 6 (1963), 163-168.

3. BROYDEN, C.G., A class of methods for solving non-linear simultaneous equations, Maths. Comp. 19, (1965), 577-593.

4. DIXON, L.C.W., Numerical methods for non-1inear optimization (1972) ed. F.A. Looft sma. Academic Press.

5. GAY, D.M., Some convergence properties of Broyden's method. SIAM. J. Num Aual $16(4),(1979), 623-630$.

6. BROYDEN, C.G., Numerical methods for non-linear algebralc equations, ed. P. Rabinowitz (1970).

7. BROYDEN, C.G., DENNIS, J.E. and MORE, J.J., On the local and superlinear convergence of Quasi-newton methods, J. Inst. Maths. Appln..12, (1973), 223245.

8. MORE, J.J. and TRANGSTEIN, J.A., On the global convergence of Broyden's method, Math. Comput. 30, (1976), 523-540.

9. DENNIS, J.R. and WALKER, H.F., Convergence theorems for least change secant update methods. SIAM J. Numerical Ana1. 18, (1981), 949-987.

10. DENNIS, J.E. and MORE, J.J., A characterization of superlinear convergence and its applications to Quasi-Newton methods. Maths. Comp. 28, (1974), 549-560.

11. DECKER, D.W. and KELLEY, C.T., Broyden's method for a class of problems having singular Jacobian at the root, SIAM J. Numer. Anal. 22(1), (1985), 566-573.

12. ORTEGA, J.M. and RHEINBOLD, W.C., Iterative solution of nonliear equations in several variables, Academic Press (1970).

13. COLlatZ, L., Functional Analysis and Numerical Analys is Ed. L. B. Rall, Academic Press, N.Y. (1966).

14. BYRNE, G.D. and HALL, C.A., Numerical solution of system of non-1inear algebraic equations, Academic Press N.Y. (1973). 


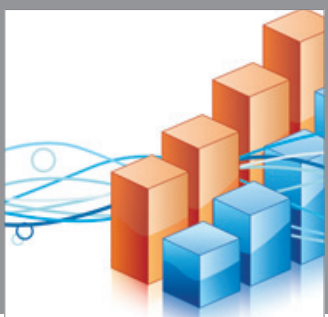

Advances in

Operations Research

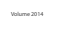

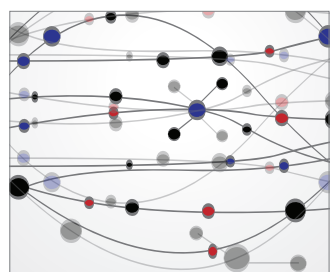

\section{The Scientific} World Journal
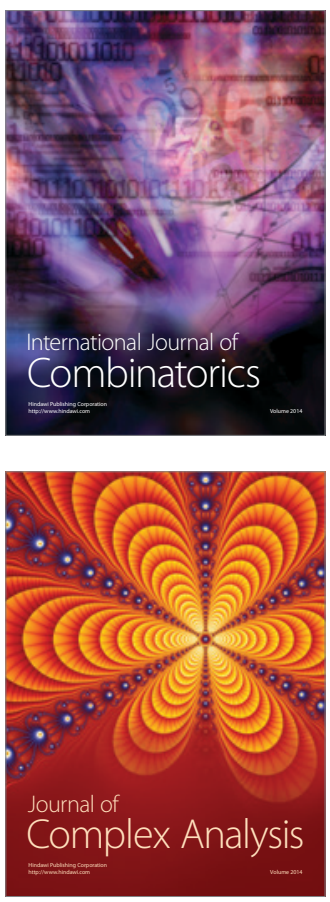

International Journal of

Mathematics and

Mathematical

Sciences
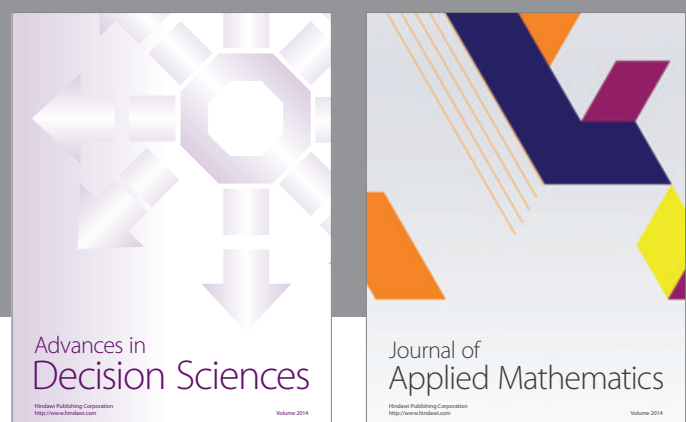

Journal of

Applied Mathematics
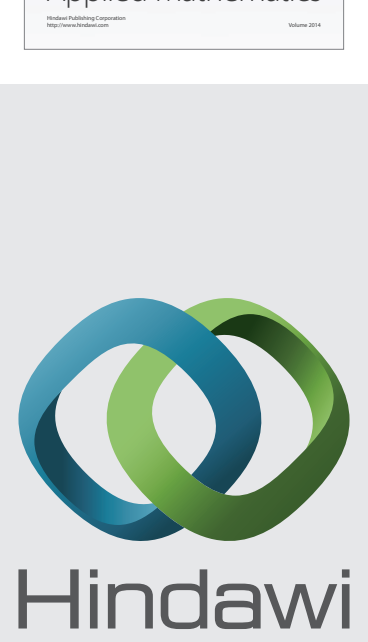

Submit your manuscripts at http://www.hindawi.com
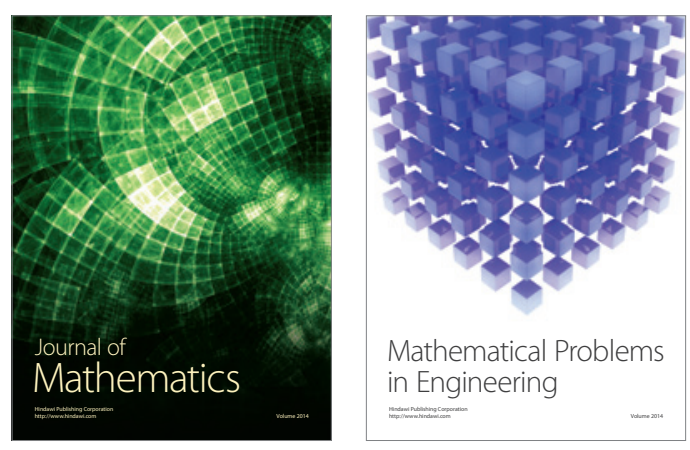

Mathematical Problems in Engineering
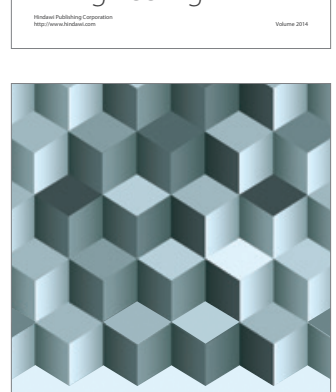

Journal of

Function Spaces
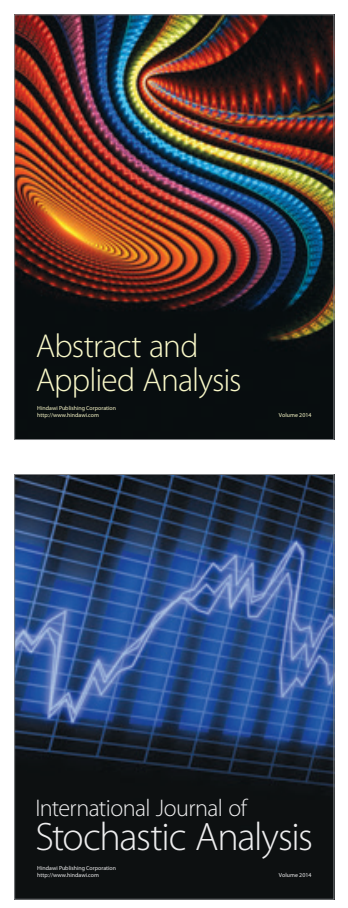

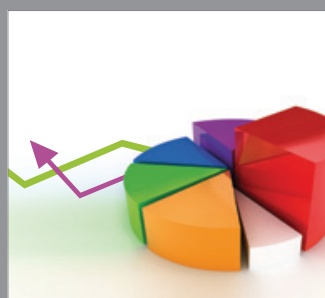

ournal of

Probability and Statistics

Promensencen
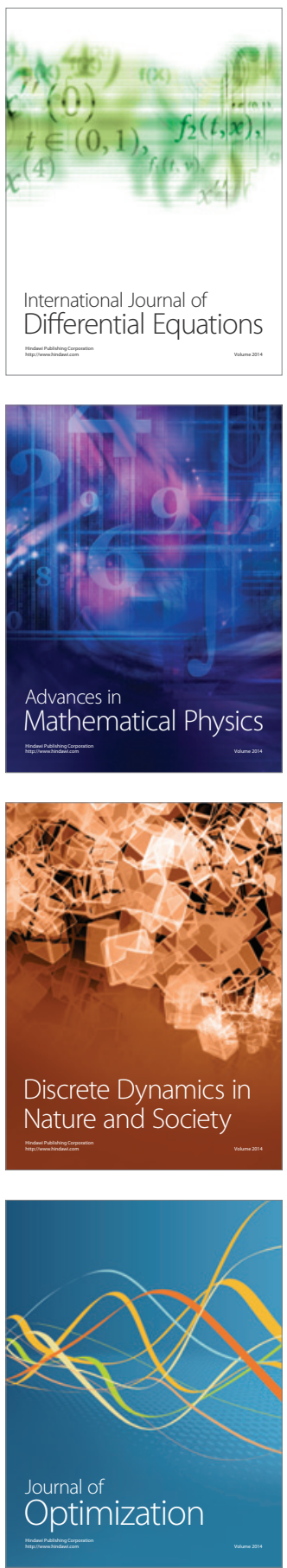